\title{
What is it to be healthy?
}

\author{
Elselijn Kingma \\ Department of History and Philosophy of Science Cambridge University Free School Lane, \\ Cambridge CB2 3RH, UK emk29@cam.ac.uk
}

\begin{abstract}
According to Christopher Boorse's Bio-Statistical Theory (BST), 'health' is statistically normal function in a reference class, and 'health' and 'disease' are empirical, objective and value-free concepts. I demonstrate that the success of the BST depends on its choice of reference classes; different reference classes result in different accounts of health. I argue that nothing in nature empirically or objectively dictates the use of reference classes Boorse proposes. Reference classes in the BST, and the concept of health, are therefore not value-free. Nor is there a reason to favour the BST over accounts of health that use different reference classes.
\end{abstract}

1.

Christopher Boorse $(1977,1997)$ argues that to be healthy is to function normally. Since normal functions are discoverable by the natural sciences, Boorse claims that health is determined solely by empirical facts and does not depend on evaluative judgement. The purpose of this paper is to show that Boorse cannot defend this claim.

According to Boorse's Bio Statistical Theory (BST), health is normal species functioning, which is the statistically typical contribution of all the organism's parts and processes to the organism's overall goals of survival and reproduction. The group with respect to which a contribution is statistically typical is the reference class, specifically an age group of a sex of a race of a species (1977: 555). This means that Tony Blair is healthy if all the parts and processes that constitute him function in ways that are statistically typical for the reference class of Caucasian, male, 53-year-old humans. Despite diverse criticisms of the BST, its appeal to reference classes is commonly accepted without question. ${ }^{1}$ This is surprising as their role in the BST is both central and dubious.

The BST needs reference classes because the human species shows a wide variety of functioning; what is normal in one group can be abnormal in another. A woman, for example, who has the level of testosterone that is normal for men, is generally considered diseased. If normal functions were those that are statistically typical for the entire species, the BST could never account for such group-specific variations in healthy function; it could not tell us that a given level of testosterone is healthy in men but a disease in women.

Therefore Boorse can only give an account of health as statistically typical functioning if he uses reference classes.

More importantly, the BST would conflict with our intuitions if reference classes other than the ones proposed by Boorse (age, sex and race) were allowed. If, for example, we were to allow a separate reference class for uncommonly heavy drinkers, then the statistically normal range for liver-functions in this group would include liver-functions normally considered a disease. The BST, however, would then entail that these liver-functions are

\footnotetext{
${ }^{1}$ An exception is Neander (1983: 92-95).
} 
normal and these heavy drinkers therefore are healthy. This example shows that the BST is only an accurate account of health if the right kind of reference classes are inserted; what it is to be healthy is not to be normal with respect to any reference class, but to be normal with respect to 'appropriate' reference classes only.

Boorse therefore needs to give an account of the distinction between reference classes that are allowed and reference classes that are not allowed. Intuitively this distinction seems clear: it makes sense to have a reference class based on age or sex, but not on being blind or having pneumonia, because the former are normal variations and the latter are diseases. This, however, is precisely what Boorse may not say. He aims to give us an account of health, and I have shown that the account he offers requires certain reference classes. If these reference classes can only be constructed based on some prior distinction between health and disease, then his account is circular. Moreover, Boorse cannot merely state which are the appropriate reference classes. Instead, since he claims to offer an account of health that is grounded in empirical fact, not evaluative judgement, he must show that empirical facts underlie the distinction between appropriate and inappropriate reference classes.

2.

Boorse proposes that an appropriate reference class is 'a natural class of organisms of uniform functional design' (1977: 562). This suggests three potential sources for a justification for treating certain reference classes as appropriate - natural, uniform and design - and I will discuss them in that order.

It is not clear what Boorse means by 'natural'. Three possible meanings spring to mind, the first of which is occurring in nature. This option must be rejected because both appropriate and inappropriate reference classes (e.g. those comprising all and only people with a certain disease) occur in nature. A second possible meaning is normal, which, if defined empirically, could be defined statistically; only sub-groups whose members occur with sufficient statistical frequency in the species as a whole are appropriate reference classes. This option must be rejected also because, although men and women occur in roughly equal proportions, some races and some age groups have very few members. Some diseases on the other hand, such as short-sightedness, are very common. If we extend our view beyond humans it is clear that there is no link between statistical frequency and reference classes: the queen-design in bees should certainly count as a reference class if anything does. It is, however, very rarely encountered.

A final possible meaning of 'natural' in this context is that reference classes are natural kinds. Assuming that natural kinds exist, and apply in biology, this solution not only requires that men and women form natural kinds, but also that races and people of different age groups do. On top of that it requires that people with Down's syndrome or people with diabetes do not form natural kinds. I cannot see how such a claim could be defended; both men and people with Down's syndrome, for example, can be identified by superficial characteristics caused by a genetic structure. ${ }^{2} \mathrm{~A}$ justification for reference classes that relies on natural kinds must therefore show that some natural kinds are the right natural kinds, whereas other natural kinds are the wrong natural kinds. But if such a justification can be provided, it goes beyond the notion of natural kinds and brings us back at the starting point of our justificatory question: why are certain reference groups appropriate but not others? An appeal to what is natural fails to answer this question.

\footnotetext{
${ }^{2}$ See Reznek (1987: 174-82) for an argument that certain disease entities are natural kinds
} 
Uniformity may fare better. The members of each of the reference classes Boorse proposes are indeed remarkably similar to each other and my anatomical atlas displays many similarities and dissimilarities that distinguish men and women. Unfortunately my atlas of pathology equally displays the (dis)similarities that enable doctors to diagnose and classify diseases. Potential reference classes that we do not want can also be remarkably uniform. Genetic syndromes, for example, are easily recognised. An appeal to uniformity therefore also fails to provide the demarcation that Boorse requires.

Boorse's last option is design. One may feel that males and females instantiate alternative designs for the human species, while the state of having pneumonia, for example, does not instantiate any such 'designed' variant form. But the term 'design' needs cashing out. Naively, one might claim that a trait counts as 'designed' in an individual if and only if the individual has the trait innately. But an appeal to innateness will not suffice since, even if we can make sense of a distinction between innate and acquired, certain (genetic) diseases are certainly innate. At the same time some traits that define appropriate reference classes, such as different ages, are in a way acquired. Some animals even acquire their sex (Charnov \& Bull 1977). If we set aside other difficulties and take the simplistic view that design is what is written in our genes, the desired distinction will not be generated either. Masculinity, Down's syndrome and Huntington's disease are all written in the genes, and the complex genetic mix I share with other Caucasians may be no more or less uniform then an equally complex mix of genes that could predispose me to diabetes, and that I would share with other diabetics.

A final interpretation of design as Nature's intent is closely related to 'natural'. This should capture the idea that Nature intended there to be men and women, but it did not intend blind people. The latter are an accident, perhaps a 'freak of nature'. Since I, and I suspect Boorse, reject an appeal to intelligent creation, the most obvious place to justify an appeal to design or Nature's intent is evolutionary biology. This is not an attractive position for Boorse, however, who explicitly rejects the idea that evolution is relevant to physiological function and health (1976: 85). To evaluate this solution in detail goes beyond the scope of this paper, but if, as I suspect it must, this solution appeals to a difference between polymorphisms that are maintained by natural selection, such as eye-colour and sexdifferences, and polymorphisms that are not maintained by natural selection, such as heartdefects, then it must at the very least dispose of the following problem. It must give a nonquestion begging account that explains why certain traits that are maintained by natural selection, such as sickle cell-anaemia, are nevertheless diseases. Since natural selection can enter into the explanation of both diseased and healthy traits (Sober 1980), this seems neither easy nor obvious, and it certainly leaves a large gap in Boorse's account. In summary, then, neither natural nor uniform nor design seem to provide Boorse with an objective justification for his selection of reference classes.

\section{3.}

When Boorse claims that the BST is a value-free account of health, he claims that the distinction between health and disease is determined by empirical facts alone. I have shown that the distinction Boorse draws between health and disease depends on counting only certain reference classes as appropriate. Different reference classes would result in different distinctions. I have also shown that Boorse gives no empirical justification for using the reference classes he proposes rather than others; although facts determine both that I am a woman and that I am short-sighted, there are no empirical facts that determine that 'women' is an appropriate reference class, and 'short sighted people' is not. Because the choice of reference classes determines the distinction between health and disease on the BST, and Boorse gives no empirical fact that justifies the choice of these reference classes over others, 
there is no empirical fact that determines the distinction between health and disease on his account. The BST therefore fails to be an empirical or value-free account of health.

Boorse would, I suspect, have a reply to this: ${ }^{3}$ he would contend that his proposed reference classes simply are the reference classes that are relevant for the distinction between health and disease. Different reference classes would generate different distinctions, but those are not the distinctions between health and disease. Although medicine might have chosen to engage with other distinctions and other concepts, this is only to say that medicine might have concerned itself with things other than health and disease. This does not make the distinction between health and disease evaluative. As he puts the point, "[t]o choose wood over concrete to build your house with is an evaluative choice, but that does not make the concepts of wood and concrete value-laden' (1997: 27).

I am happy to accept Boorse's reply, but it does not block the implications my analysis has for his account. Boorse offers an account of health that is supposed to tell us whether a condition, for example homosexuality (to take a contested example) is healthy or a disease. Boorse also claims that his account gives an answer to this question that is value-free, which is his main selling point. Let me now phrase this question in a different way: imagine there are two candidate concepts for health. One is the BST, and one is the XST. The XST is exactly like the BST, but has one more reference class: sexual orientation. Thus on the XST homosexuality is a normal, therefore healthy, function in the reference class of homosexual people. On the BST however, homosexuality interferes with statistically typical reproductive function in the reference class of all men, and is therefore a disease. The question 'is homosexuality a disease?' then reduces to the question 'is the BST or the XST the right account of health?', or, 'is sexual orientation an appropriate reference class or not?'. Since, as I have argued, there are no facts that determine which reference classes are appropriate, there is no empirical fact that determines whether homosexuality is an appropriate reference class. Therefore there is no empirical fact that tells us whether the BST or the XST is correct.

Since the judgement that homosexuality is a disease precedes the judgement that the BST is a correct account of health, it is circular to insist, as Boorse does, that the BST can tell us whether homosexuality is a disease. ${ }^{4}$ The BST does not give a real answer, let alone a nonevaluative answer, to such a question. Instead it presupposes the answer it gives, and Boorse must adopt a more modest claim: once the reference classes are fixed the BST gives an accurate and value-free analysis of health and disease. In other words, once reference classes are fixed the BST does not appeal to social judgements to move from the facts about a case to a judgement about its health status. The fixing of reference classes, however, is an evaluative choice which may reflect some deep underlying normative commitments to, for example, ideas about normal sexual attraction. Therefore I must still conclude that the BST is in all relevant ways evaluative, and that Boorse can no longer defend what he claims is the main benefit of his account: that the BST is value-free. ${ }^{5}$

\section{References}

Boorse C. On the distinction between disease and illness. Philosophy of Public Affairs. 1975; 5:49-68.

Boorse C. Wright on functions. The Philosophical Review. 1976; 85:70-86.

Boorse C. Health as a theoretical concept. Philosophy of Science. 1977; 44:542-73.

\footnotetext{
${ }^{3}$ I model Boorse's reply on his response to the objection that his choice of goals is normative. See 1997: 25-28.

${ }^{4}$ Boorse defends the view that homosexuality is a disease. However, as he repeatedly argues, this does not entail that homosexuality is either a bad thing or ought to be treated (1975: 63 \& 1997: 99).

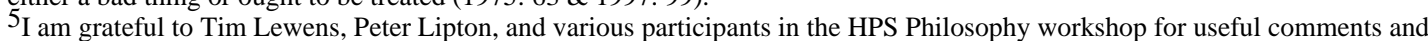
suggestions, and to the Wellcome Trust, grant number 077887, for generous financial assistance.
} 
Boorse, C. A rebuttal on health. In: Humber, JM.; Almeder, RF., editors. What is Disease?. Totowa, New Jersey: Humana Press; 1997.

Charnov E, Bull J. When is sex environmentally determined? Nature. 1977; 266:828-30. [PubMed: 865602]

Neander, K. Abnormal Psychobiology. La Trobe University; 1983. PhD dissertation

Reznek, L. The Nature of Disease. London: Routledge and Kegan Paul; 1987.

Sober E. Evolution, population thinking and essentialism. Philosophy of Science. 1980; 47:350-83. 